\title{
Echoentomography for Assessing Braconid Parasitization on Soft-Bodied Tephritid Hosts
}

\author{
Renato Ricciardi ${ }^{1} \mathbb{1}$, Rossana Izzetti ${ }^{2}$, Marco Romanelli ${ }^{3}$, Davide Caramella ${ }^{4}$, Andrea Lucchi ${ }^{1}$ \\ and Giovanni Benelli $1, *$ (D) \\ 1 Department of Agriculture, Food and Environment, University of Pisa, Via del Borghetto 80, 56124 Pisa, Italy; \\ renato_ricciardi@hotmail.it (R.R.); andrea.lucchi@unipi.it (A.L.) \\ 2 Unit of Dentistry and Oral Surgery, Department of Surgical, Medical and Molecular Pathology and Critical \\ Care Medicine, University of Pisa, 56124 Pisa, Italy; ross.izzetti@gmail.com \\ 3 Unit of Dermatology, Department of Clinical and Experimental Medicine, University of Pisa, 56124 Pisa, Italy; \\ marco.romanelli@unipi.it \\ 4 Diagnostic and Interventional Radiology, Department of Translational Research and of New Technologies in \\ Medicine and Surgery, University of Pisa, 56124 Pisa, Italy; davide.caramella@unipi.it \\ * Correspondence: giovanni.benelli@unipi.it
}

Citation: Ricciardi, R.; Izzetti, R.; Romanelli, M.; Caramella, D.; Lucchi, A.; Benelli, G. Echoentomography for Assessing Braconid Parasitization on Soft-Bodied Tephritid Hosts. Insects 2021, 12, 980. https://doi.org/ $10.3390 /$ insects 12110980

Academic Editors: Andrea Di Giulio and Roberto Romani

Received: 6 October 2021

Accepted: 25 October 2021

Published: 29 October 2021

Publisher's Note: MDPI stays neutral with regard to jurisdictional claims in published maps and institutional affiliations.

Copyright: (c) 2021 by the authors. Licensee MDPI, Basel, Switzerland. This article is an open access article distributed under the terms and conditions of the Creative Commons Attribution (CC BY) license (https:/ / creativecommons.org/licenses/by/ $4.0 /)$.
Simple Summary: Host parasitization by an endoparasitoid insect can be evaluated relying on three main methods, i.e., dissection under a stereomicroscope, polymerase chain reaction (PCR), or waiting for adult emergence. These approaches show limitations related to the time required for the detection of parasitization and/or the destruction of the sample. In this research, an innovative approach using ultra-high frequency ultrasound (UHFUS) technology was employed to evaluate braconid parasitization of Ceratitis capitata larvae. The UHFUS approach was compared with classic stereomicroscopic dissection, showing that both methods provide comparable diagnostic reliability. Our results support the application of echoentomography as a useful tool for fine, fast, and noninvasive evaluation of the ability of parasitoids to parasitize soft-bodied hosts.

Abstract: Entomological approaches currently available for assessing host parasitization require dissection, polymerase chain reaction (PCR), or waiting for adult emergence. The first two methods are relatively fast but destructive, whereas the third one allows the emergence of the parasitoid but it is time consuming. In this framework, new diagnostic imaging tools may contribute to solve the lack of an accurate, rapid, and non-invasive approach to evaluate the parasitization of soft-bodied insects by their endoparasitoids. In this study, ultra-high frequency ultrasound (UHFUS) technology, which is currently used in medical and preclinical fields, was adopted to assess the parasitization of the invasive polyphagous Mediterranean fruit fly, Ceratitis capitata (Diptera: Tephritidae), testing 2nd and 3rd instar larvae. Parasitization assays were carried out with the solitary koinobiont endophagous parasitoid Psyttalia concolor (Hymenoptera: Braconidae: Opiinae). The efficacy of UHFUS-based echoentomography was compared with the classical method of dissecting the larval host under a stereomicroscope. Our results showed that the UHFUS diagnostic capability was statistically comparable with that of dissection, both on C. capitata 2nd and 3rd larvae. Overall, UHFUS-based echoentomography may be further considered as a fast, non-invasive, and effective approach to evaluate the parasitoid's ability to successfully oviposit in soft-bodied hosts.

Keywords: Braconidae; Ceratitis capitata; insect imaging; parasitoid mass rearing; Psyttalia concolor; Tephritidae; ultra-high frequency ultrasound (UHFUS) technology

\section{Introduction}

Recently, technologies such as radiography, computed tomography (CT) and micro-CT, magnetic resonance imaging (MRI), and ultrasonography, which were previously strictly dedicated to medical and pre-clinical research, have been considered for studying insect 
morpho-physiology, achieving fascinating results [1-3]. Indeed, these studies gave detailed insights about the functional anatomy of the insect's brain [4,5], muscles [1], exoskeleton, and respiratory system [3].

Assessing the parasitization success of mass-reared hymenopteran parasitoids on their potential hosts is of crucial importance for developing inexpensive and reliable large-scale production of biological control agents (BCA) [6,7]. Current methods to detect parasitization efficacy on a given insect host are time-consuming and/or destructive, mainly based on waiting for the parasitoid emergence, if the parasitoid's egg is not encapsulated by the host's immune system, on anatomical dissection of the host under the stereomicroscope [8], and on polymerase chain reaction (PCR) to detect and identify parasitoid DNA [9,10]. In this framework, fast-track non-invasive methods are needed, with special reference to rearing of rare or hard-to-maintain species of potential biocontrol relevance.

The present study aims to provide a novel fast-track approach, allowing real-time detection of successful parasitoid oviposition on a given host, relying on ultra-high frequencies ultrasound (UHFUS) technology. This is a novel implementation of the conventional ultrasound technology, allowing visualization of tiny anatomical details, thanks to advanced UHF transducers, to reach outstanding spatial resolution at frequencies up to $100 \mathrm{MHz}[2,11-14]$. As a study model, we focused on the trophic interaction between the endoparasitoid Psyttalia concolor (Szépligeti) (Hymenoptera: Braconidae) and its host, the Mediterranean fruit fly (medfly), Ceratitis capitata (Wiedemann) (Diptera: Tephritidae), an invasive polyphagous species able to attack over 250 plant species [15]. Psyttalia concolor is a synovigenic koinobiont larval-pupal endoparasitoid. This solitary parasitic wasp attacks 2nd-3rd instar larvae of at least fourteen tephritid species [16], including the medfly and the olive fruit fly, Bactrocera oleae (Rossi) (Diptera: Tephritidae) [16]. To cope with the above-mentioned challenges, herein a non-invasive UHFUS-based approach allowing real-time detection of the parasitoid's egg in a living host was proposed, assessing the oviposition success of $P$. concolor in C. capitata larvae. The reliability of UHFUS-based echoentomography was compared with that of traditional invasive dissection under a stereomicroscope.

\section{Materials and Methods}

\subsection{Insect Rearing}

Ceratitis capitata adults were mass-reared in cylindrical PVC cages $(\varnothing=35 \mathrm{~cm} ; \mathrm{h}=35 \mathrm{~cm})$ at a density of about 2000 flies per cage. Medflies were fed on a dry diet composed by sucrose and yeast extract (Sigma-Aldrich, Germany) (10:1, w:w); water was provided on a cotton wick through dedicated dispensers. Larvae were reared in plastic bowls $(50 \times 15 \times 2 \mathrm{~cm})$ containing $0.5 \mathrm{~kg}$ of an artificial culture medium (i.e., bran, devitalized yeast, wheat germ, and sugar), as detailed by Canale and Benelli [16]. Our medfly rearing is maintained in laboratory conditions at $23 \pm 2{ }^{\circ} \mathrm{C}$ and relative humidity (RH) of $45 \pm 5 \%$.

Psyttalia concolor young instars were reared on 3rd instar medfly larvae, according to Canale and Benelli [16]. Psyttalia concolor adults were fed ad libitum on a mixture of honey and bee-collected pollen in small cylindrical vial caps $(0.5 \times 2 \mathrm{~cm})$. Water was provided separately on a cotton wick [17].

\subsection{Host Parasitization Protocol and UHFUS Observations}

Using the multiple-well arena described by Benelli et al. [17], covered with a transparent Petri dish lid $(\varnothing=55 \mathrm{~mm} ; \mathrm{h}=13 \mathrm{~mm})$ (Figure 1), forty 2 nd and 3rd instar C. capitata larvae were parasitized by P. concolor females. Second and third instar medfly larvae were empirically identified according to their size and the number of days since the eggs hatched at a known temperature [16]. Each medfly larva was parasitized individually by a $P$. concolor female released in the above-mentioned arena; each larva was considered successfully parasitized when the parasitoid kept the ovipositor in the host for at least $20 \mathrm{~s} \mathrm{[18];} \mathrm{for} \mathrm{this} \mathrm{reason,} \mathrm{the} \mathrm{host's} \mathrm{exposure} \mathrm{to} \mathrm{the} \mathrm{parasitoid} \mathrm{was} \mathrm{directly} \mathrm{monitored} \mathrm{by}$ an observer. 


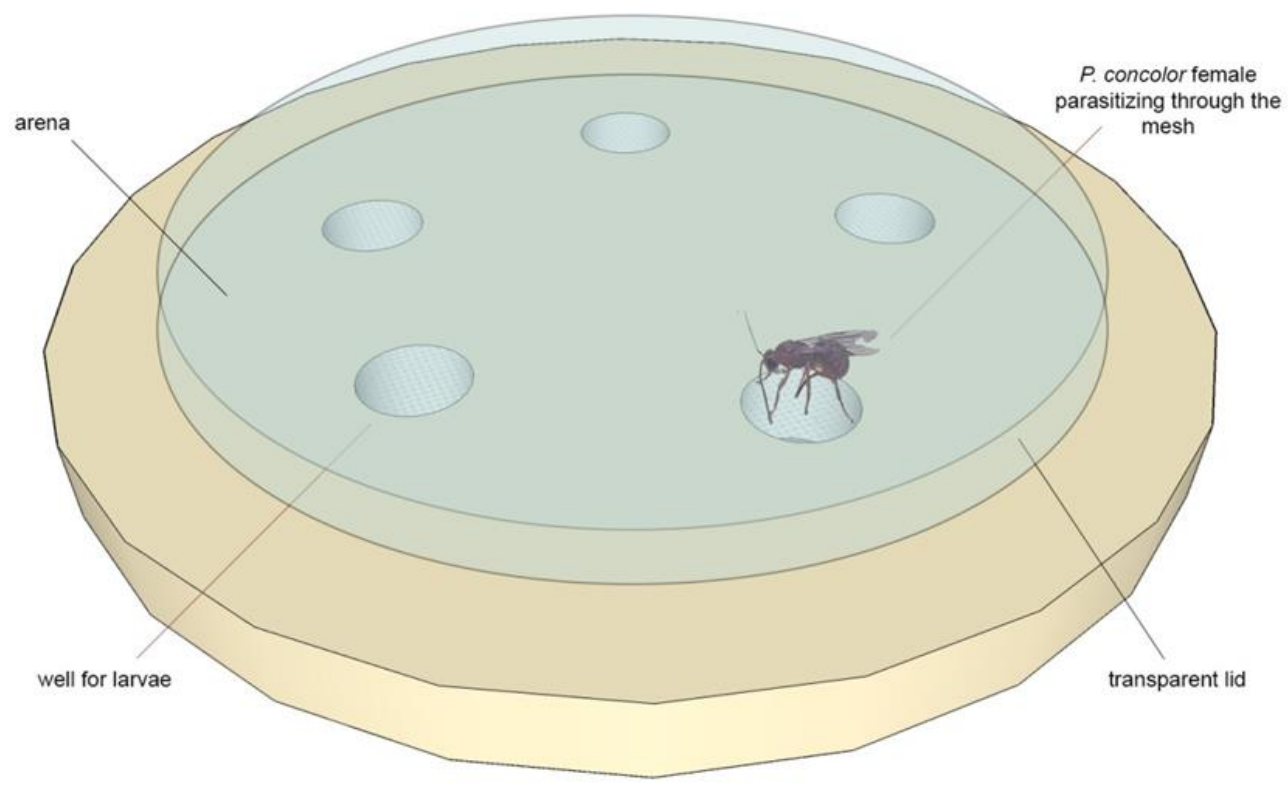

Figure 1. A schematic representation of the arena proposed by Benelli et al. [17] for tephritid parasitization by braconid larval-pupal endoparasitoids; each well was filled with a single larva plus its rearing medium. Then, a parasitoid female was allowed to parasitize the larva through the mesh holes covering the wells, mimicking the fruit's surface.

For each selected instar, 40 larvae were analyzed through UHFUS in vivo observations using the Vevo ${ }^{\circledR} \mathrm{MD}$ (Fujifilm, VisualSonics, Toronto, ON, Canada) $70 \mathrm{MHz}$ transducer, and following the method adopted by Ricciardi et al. [2]. The $70 \mathrm{MHz}$ transducer (UHF 70) is characterized by a $30 \mu$ axial resolution, which makes it suitable for the investigation of structures of extremely small size. Therefore, plastic wells slightly larger than the larvae $(8.4 \times 2.6 \times 5 \mathrm{~mm})$ were used to minimize larvae movements without blocking them. The probe was locked to an adjustable support, $2-3 \mathrm{~mm}$ above the study insect included in sterile ultrasound gel (Teleflex Medical, Varedo, Italy); 2D mode scans were carried out. After each analysis, the larvae were gently washed with distilled water and separated into vials to allow parasitoid development. The remaining 40 larvae, representing the control, were routinely dissected in 70\% ethanol under the stereomicroscope (Greenough S9e, Leica Microsystems, Wetzlar, Germany) searching for P. concolor eggs. All trials were conducted from July to September 2020.

\subsection{Statistical Analysis}

The reliability of UHFUS-based echoentomography in detecting a successful parasitoid oviposition (estimated in terms of egg no. detected in each medfly host) was compared with classic dissection using a contingency analysis. JMP 11 (SAS) was used for the analyses.

\section{Results}

Relying on the UHFUS technology, it was possible to observe the presence of one (Figure 2A) or more P. concolor eggs (Figure 2B) within the medfly host, thus confirming the parasitization event. The analysis of each sample required a short time; indeed, within 3 to $5 \mathrm{~min}$ it was possible to assess the presence or absence of parasitoid eggs (laid $6 \mathrm{~h}$ before) in the $C$. capitata larval body. The eggs appear slightly curved in shape, with a whitish nucleus immersed in cytosol (black area surrounding the core), all contained by the chorion. 

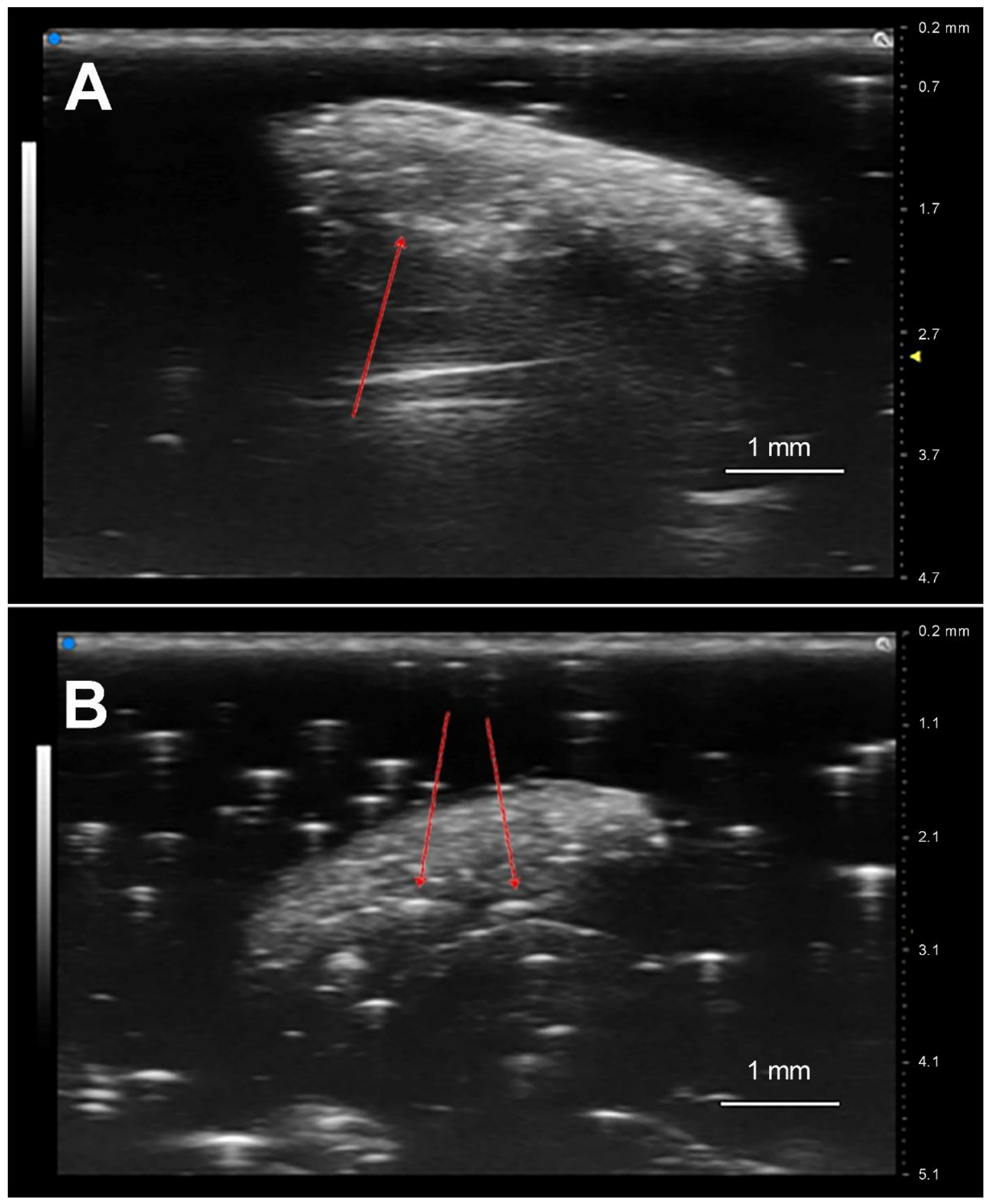

Figure 2. Ultrasound scans, $6 \mathrm{~h}$ after parasitization, showing the presence of (A) an egg of the braconid parasitoid Psyttalia concolor in a 3rd instar of Ceratitis capitata and (B) two eggs of the same parasitoid in a 2nd instar medfly larva.

Microscopic dissection of the parasitized medfly larvae confirmed the gross morphology of the braconid parasitoid egg, as observed through UHFUS (Figure 3). Comparing the two techniques to evaluate successful parasitization of second $\left(\chi^{2}=3.55, d . f .=1, p=0.059\right)$ and third $\left(\chi^{2}=0.675\right.$, d.f. $\left.=1, p=0.411\right)$ instar medfly larvae, no significant difference was noted between the diagnostic accuracy of the two techniques (Figure 4A,B). Moreover, through UHFUS-based echoentomography, it was possible to assess, quite simply, the size of the eggs, corresponding to $0.49 \pm 0.02 \mathrm{~mm}$ (length, mean $\pm \mathrm{SD}$ ). 


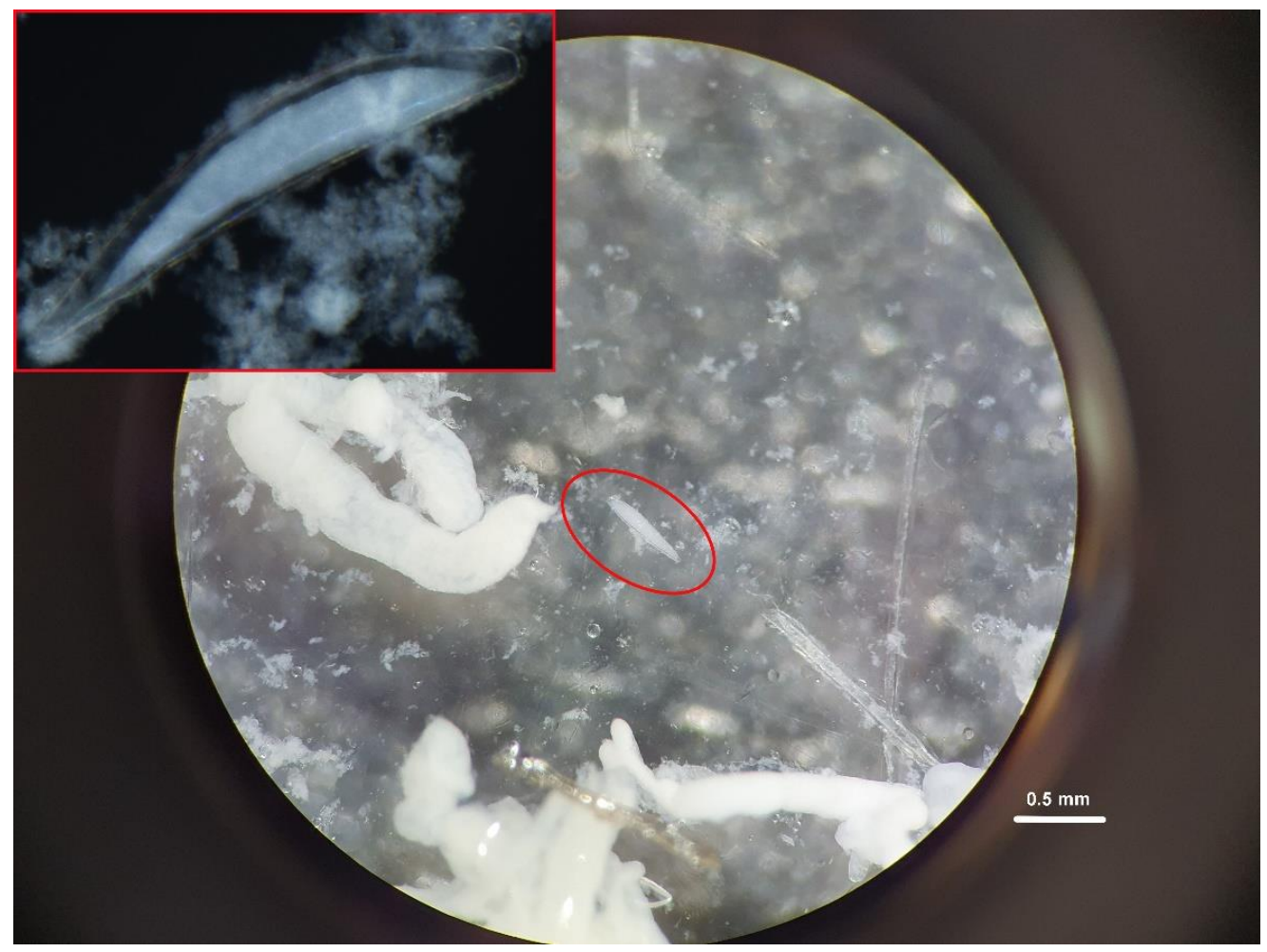

Figure 3. Eggs of Psyttalia concolor (marked by red circle) detected through dissection in $70 \%$ ethanol of a 2 nd instar of Ceratitis capitata, $6 \mathrm{~h}$ after parasitization. In the left corner of the figure, an enlarged view of the parasitoid egg.

(A)

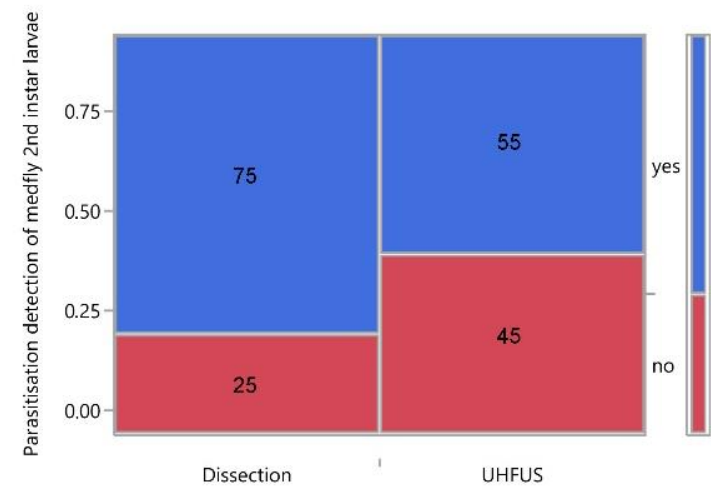

(B)

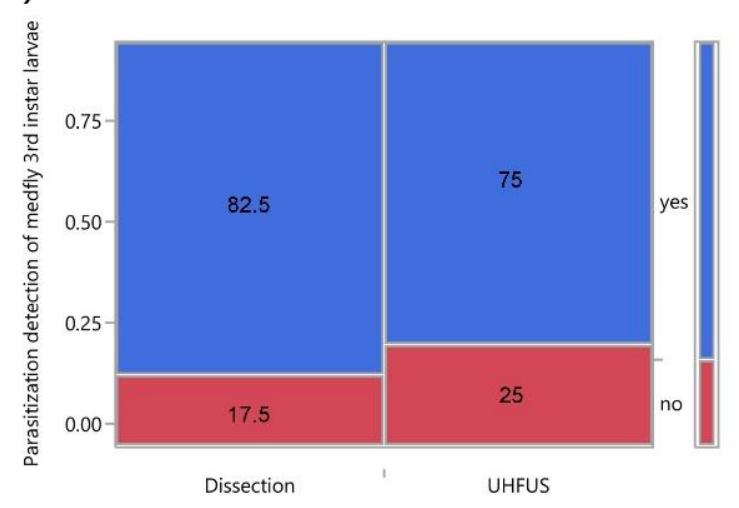

Figure 4. Contingency analysis comparing the diagnostic efficacy of ultrasonography (UHFUS) and stereomicroscopic dissection carried out on (A) 2nd and (B) 3rd instar larvae of Ceratitis capitata parasitized $6 \mathrm{~h}$ before by Psyttalia concolor. The bar on the right represents the relative abundance of successful parasitization events compared with the total number of tested medfly larvae (blue-yes, indicates the larvae where one or more eggs of the parasitoid have been detected; red-no, indicates the larvae where no eggs of the parasitoid have been detected). The number in each box indicates the percentage of parasitized medfly larvae.

\section{Discussion}

Currently, UHFUS technology is mainly used in the medical field, in particular for the evaluation of cutaneous structures, which makes the technique extremely performing in dermatology and oral medicine $[19,20]$. However, in recent years it has been employed for preclinical studies on model animals such as zebrafish [21] and rodents [22,23]. Of note, high-frequency ultrasound-based approaches can be of interest for studying soft-bodied 
insects in vivo, leading to a novel research approach recently named as echoentomography [2]. On the other hand, insects characterized by a solid chitinous exoskeleton are poorly suitable for this kind of investigation due to the difference in acoustic impedance that causes the reflection of most waves at the boundary between gel and their exoskeleton, not allowing a clear analysis of the tissues. In the case of soft-bodied insects, UHFUS-based echoentomography can allow the observation of the morpho-physiology of economically important insect species, simultaneously preserving the viability of the individual. For example, a recent study relying on UHFUS-based echoentomography allowed the in vivo analysis of C. capitata and Lobesia botrana (Denis \& Schiffermüller) (Lepidoptera: Tortricidae) larvae, allowing the quantification key anatomical and physiological features, such as the pyloric valve rotating movements and haemolymph flux, just to cite some [2].

In our opinion, UHFUS-based echoentomography may also be used for real-time studies to assess the impact of biotic and abiotic stressors, such as selected insecticides used to control the soft-bodied juvenile stages of a given pest species. Furthermore, investigations of host-parasite interactions could be carried out using this approach. On this last assumption, herein we compared the reliability of echoentomography and stereomicroscopic dissection, to detect the eggs laid by P. concolor females in the soft body of C. capitata 2 nd and 3rd instar. Our results revealed the possibility of assessing the host parasitization in vivo with an accuracy comparable with stereomicroscopic dissection, and a faster processing time for each tested individual.

Currently, three main tools are used to assess the potential successful parasitization of a target insect: (i) waiting for the parasitoid emergence from the host [24,25], (ii) dissecting the host to assess the presence of host eggs [26,27], or (iii) using PCR to detect and identify parasitoid DNA $[9,10]$. The first approach is inexpensive and not destructive, but slow since parasitization can be confirmed only after several days or weeks. The second approach is equally inexpensive, parasitization can be quickly detected, but the overall analysis is invasive for both the involved individuals. The PCR-based approach is efficient, but destructive and more expensive compared with the above-mentioned ones [28]. Compared with these approaches, UHFUS-based echoentomography is rapid, effective, non-invasive, and allows in vivo analysis. However, the latter is limited to observations on the larval stage of the host, because the study of hard-bodied insects or chitinous structures such as the pupa proved to be impossible, due to the total reflection of the ultrasound wave by the exoskeleton [2]. Moreover, it is also inexpensive when performed on equipment already available in the clinical setting, which can be accessible for non-clinical research at little or no additional costs. Indeed, the introduction of UHFUS for clinical use is very recent, and medical applications and clinical research are not able to saturate the equipment yet, making it freely available for non-clinical research.

It should be noted that the exclusive use of UHFUS for entomological purposes may not be justified if the volume of examinations is limited, due to the high acquisition and maintenance costs of the equipment. As a matter of fact, the cost of dissecting microscopes is much lower when compared with UHFUS. Nevertheless, the distinct advantage provided by UHFUS in terms of reduced invasiveness and the possibility to perform in vivo analysis appears very promising, and we foresee a growing application of the technique in entomology. Therefore, establishing a collaboration between research groups and clinical centers already equipped with UHFUS may help to overcome such a limitation.

\section{Conclusions and Challenges for Future Research}

Overall, relying on UHFUS-based echoentomography for assessing successful parasitization of soft-bodied hosts by hymenopteran endoparasitoids allows rapid, reliable, and non-invasive assessment of the parasitization success during the larval phases of the host. Moreover, the proposed methodology may represent a valuable tool to finely track the development and the morpho-physiology of parasitoid preimaginal stages developing in soft-bodied larval hosts. The opportunity to analyze and monitor insect morpho-physiology in vivo may have an important application value, allowing the non- 
invasive study of difficult-to-collect or endangered species, as well as the investigation of key lethal and sub-lethal effects due to insecticide exposure.

Author Contributions: Conceptualization, R.R., D.C., A.L. and G.B.; methodology, R.R., R.I., M.R., D.C. and G.B.; software, M.R. and D.C.; validation, R.R., R.I., M.R., D.C., A.L. and G.B.; formal analysis, R.R., R.I., M.R., D.C., A.L. and G.B.; investigation, R.R., R.I. and G.B.; resources, M.R., D.C. and A.L.; data curation, R.R. and G.B.; writing-original draft preparation, R.R. and G.B.; writing-review and editing, R.R., R.I., M.R., D.C., A.L. and G.B.; visualization, R.I., M.R., D.C., A.L. and G.B.; supervision, M.R., D.C., A.L. and G.B.; project administration, M.R., D.C. and A.L.; funding acquisition, M.R., D.C., A.L. and G.B. All authors have read and agreed to the published version of the manuscript.

Funding: This research received no external funding.

Institutional Review Board Statement: Not applicable.

Informed Consent Statement: Not applicable.

Data Availability Statement: Data are contained within the article.

Acknowledgments: The authors are grateful to Marzia Coppola (University of Pisa) for her kind assistance during UHFUS experiments, and to Augusto Loni (University of Pisa) for his kind help in P. concolor rearing.

Conflicts of Interest: The authors declare no conflict of interest.

\section{References}

1. Li, D.; Zhang, K.; Zhu, P.; Wu, Z.; Zhou, H. 3D configuration of mandibles and controlling muscles in rove beetles based on micro-CT technique. Anal. Bioanal. Chem. 2011, 401, 817-825. [CrossRef] [PubMed]

2. Ricciardi, R.; Aringhieri, G.; Faita, F.; Benelli, G.; Boccaccio, C.; Lucchi, A.; Caramella, D. Echoentomography: A novel nondestructive imaging of soft-body insects through ultra-high frequency ultrasonography (UHFUS). Entomol. Gen. 2021. [CrossRef]

3. Shaha, R.K.; Vogt, J.R.; Han, C.-S.; Dillon, M.E. A micro-CT approach for determination of insect respiratory volume. Arthropod Struct. Dev. 2013, 42, 437-442. [CrossRef]

4. Ribi, W.; Senden, T.J.; Sakellariou, A.; Limaye, A.; Zhang, S. Imaging honey bee brain anatomy with micro-X-ray-computed tomography. J. Neurosci. Methods 2008, 171, 93-97. [CrossRef] [PubMed]

5. Smith, D.B.; Bernhardt, G.; Raine, N.E.; Abel, R.L.; Sykes, D.; Ahmed, F.; Pedroso, I.; Gill, R.J. Exploring miniature insect brains using micro-CT scanning techniques. Sci. Rep. 2016, 6, 21768. [CrossRef] [PubMed]

6. Bautista, R.C.; Mochizuki, N.; Spencer, J.P.; Harris, E.J.; Ichimura, D.M. Mass-rearing of the tephritid fruit fly parasitoid Fopius arisanus (Hymenoptera: Braconidae). Biol. Control 1999, 15, 137-144. [CrossRef]

7. Parra, J.R.P. Mass rearing of egg parasitoids for biological control programs. In Egg Parasitoids in Agroecosystems with Emphasis on Trichogramma; Springer: Berlin/Heidelberg, Germany, 2009; pp. 267-292.

8. Day, W.H. Estimating mortality caused by parasites and diseases of insects: Comparisons of the dissection and rearing methods. Environ. Entomol. 1994, 23, 543-550. [CrossRef]

9. Ashfaq, M.; Shah, G.S.; Noor, A.R.; Ansari, S.P.; Mansoor, S. Report of a parasitic wasp (Hymenoptera: Encyrtidae) parasitizing cotton mealybug (Hemiptera: Pseudococcidae) in Pakistan and use of PCR for estimating parasitism levels. Biocontrol Sci. Technol. 2010, 20, 625-630. [CrossRef]

10. Gariepy, T.D.; Kuhlmann, U.; Gillott, C.; Erlandson, M. Parasitoids, predators and PCR: The use of diagnostic molecular markers in biological control of Arthropods. J. Appl. Entomol. 2007, 131, 225-240. [CrossRef]

11. Albano, D.; Messina, C.; Usuelli, F.G.; De Girolamo, L.; Grassi, M.; Maccario, C.; Bignotti, B.; Tagliafico, A.; Sconfienza, L.M. Magnetic resonance and ultrasound in Achilles tendinopathy: Predictive role and response assessment to platelet-rich plasma and adipose-derived stromal vascular fraction injection. Eur. J. Radiol. 2017, 95, 130-135. [CrossRef] [PubMed]

12. Brown, J.M.; Yablon, C.M.; Morag, Y.; Brandon, C.J.; Jacobson, J.A. US of the peripheral nerves of the upper extremity: A landmark approach. Radiographics 2016, 36, 452-463. [CrossRef]

13. Izzetti, R.; Vitali, S.; Oranges, T.; Dini, V.; Romanelli, M.; Caramella, D.; Gabriele, M. Intraoral Ultra-High Frequency Ultrasound study of oral lichen planus: A pictorial review. Ski. Res. Technol. 2020, 26, 200-204. [CrossRef] [PubMed]

14. Sokolov, S.J. Ultrasonic oscillations and their applications. Tech. Phys. USSR 1935, 2, 522-534.

15. White, I.M.; Elson-Harris, M.M. Fruit Flies of Economic Significance: Their Identification and Bionomics; CAB International: Wallingford, UK, 1992; ISBN 0851987907.

16. Benelli, G.; Canale, A. Learning of visual cues in the fruit fly parasitoid Psyttalia concolor (Szépligeti) (Hymenoptera: Braconidae). BioControl 2012, 57, 767-777. [CrossRef]

17. Benelli, G.; Gennari, G.; Canale, A. Host discrimination ability in the tephritid parasitoid Psyttalia concolor (Hymenoptera: Braconidae). J. Pest Sci. 2013, 86, 245-251. [CrossRef] 
18. Canale, A.; Benelli, G. Impact of mass-rearing on the host seeking behaviour and parasitism by the fruit fly parasitoid Psyttalia concolor (Szépligeti) (Hymenoptera: Braconidae). J. Pest Sci. 2012, 85, 65-74. [CrossRef]

19. Berritto, D.; Iacobellis, F.; Rossi, C.; Reginelli, A.; Cappabianca, S.; Grassi, R. Ultra high-frequency ultrasound: New capabilities for nail anatomy exploration. J. Dermatol. 2017, 44, 43-46. [CrossRef]

20. Oranges, T.; Vitali, S.; Benincasa, B.; Izzetti, R.; Lencioni, R.; Caramella, D.; Romanelli, M.; Dini, V. Advanced evaluation of hidradenitis suppurativa with ultra-high frequency ultrasound: A promising tool for the diagnosis and monitoring of disease progression. Ski. Res. Technol. 2010, 26, 513-519. [CrossRef] [PubMed]

21. Sun, L.; Lien, C.-L.; Xu, X.; Shung, K.K. In vivo cardiac imaging of adult zebrafish using high frequency ultrasound (45-75 MHz). Ultrasound Med. Biol. 2008, 34, 31-39. [CrossRef]

22. Wu, G.; Wang, L.; Yu, L.; Wang, H.; Xuan, J.W. The use of three-dimensional ultrasound micro-imaging to monitor prostate tumor development in a transgenic prostate cancer mouse model. Tohoku J. Exp. Med. 2005, 207, 181-189. [CrossRef] [PubMed]

23. Yeh, C.K.; Chen, J.J.; Li, M.L.; Luh, J.J.; Chen, J.J.J. In vivo imaging of blood flow in the mouse Achilles tendon using high-frequency ultrasound. Ultrasonics 2009, 49, 226-230. [CrossRef] [PubMed]

24. Chong, J.H.; Oetting, R.D. Functional response and progeny production of the Madeira mealybug parasitoid, Anagyrus sp. nov. nr. sinope: The effects of host and parasitoid densities. Biol. Control 2006, 39, 320-328. [CrossRef]

25. Ricciardi, R.; Zeni, V.; Michelotti, D.; Di Giovanni, F.; Cosci, F.; Canale, A.; Zang, L.S.; Lucchi, A.; Benelli, G. Old parasitoids for new mealybugs: Host location behavior and parasitization efficacy of Anagyrus vladimiri on Pseudococcus comstocki. Insects 2021, 12, 257. [CrossRef] [PubMed]

26. Feng, D.-D.; Li, P.; Zhou, Z.-S.; Xu, Z.-F. Parasitism potential of Aenasius bambawalei on the invasive mealybug Phenacoccus solenopsis. Biocontrol Sci. Technol. 2014, 24, 1333-1338. [CrossRef]

27. Sagarra, L.A.; Vincent, C.; Peters, N.F.; Stewart, R.K. Effect of host density, temperature, and photoperiod on the fitness of Anagyrus kamali, a parasitoid of the hibiscus mealybug Maconellicoccus hirsutus. Entomol. Exp. Appl. 2000, 96, 141-147. [CrossRef]

28. Persad, A.B.; Jeyaprakash, A.; Hoy, M.A. High-fidelity PCR assay discriminates between immature Lipolexis oregmae and Lysiphlebus testaceipes (Hymenoptera: Aphidiidae) within their aphid hosts. Florida Entomol. 2004, 87, 18-24. [CrossRef] 